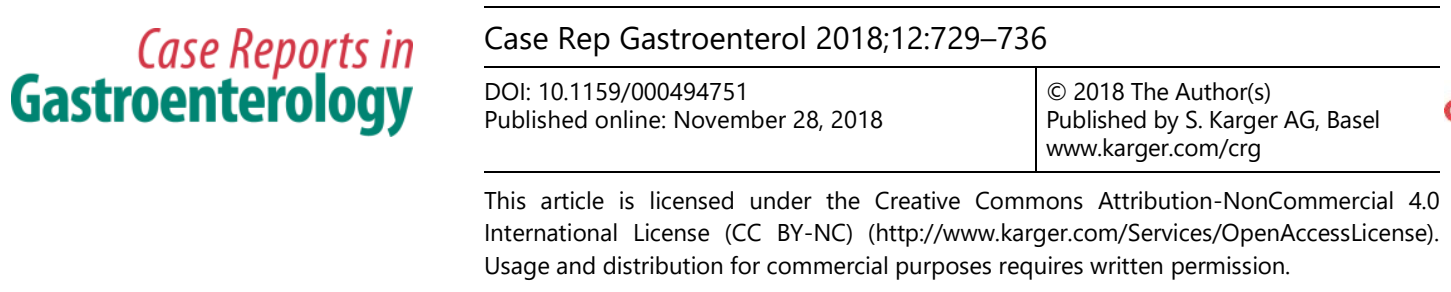

\title{
Whole Genome Sequencing Analysis of a Stage IV Colon Cancer Patient with a 10-Year Disease-Free Survival following Systemic Chemotherapy/Bevacizumab
}

\author{
Timothy J. Yeatman ${ }^{a}$ Mingli Yang ${ }^{a}$ Domenico Coppolab \\ aGibbs Cancer Center \& Research Institute, Spartanburg, SC, USA; b Department of \\ Anatomic Pathology, Moffitt Cancer Center \& Research Institute, Tampa, FL, USA
}

\section{Keywords}

Colon cancer - Genomic testing · Driver genes · Chemotherapy · Bevacizumab

\begin{abstract}
It is rare that stage IV colon cancer is cured with chemotherapy. Here we report the long-term survival of a patient who presented with highly advanced disease characterized by a papillary architecture as well as porta hepatis lymph nodes but responded extremely well to FOLFIRI/bevacizumab. His original tumor underwent comprehensive genomic testing that included whole genome DNA sequencing, targeted sequencing, and RNA sequencing. These genetic results suggest the patient's tumor harbored mutations in APC, KRAS, and TP53 as well as in PIK3CB. Moreover, the RNA-seq data suggested that the tumor belonged to the consensus molecular subtype 4, the "inflamed, immune phenotype," with increased angiogenesis. Deep sequencing of highly responsive cancers may yield molecular insights into mechanisms underpinning a remarkable response.

(c) 2018 The Author(s) Published by S. Karger AG, Basel
\end{abstract}

\section{Introduction}

Colorectal cancer (CRC) is a deadly disease, particularly when patients present with stage IV, metastatic disease [1]. Improved use of biologic agents, in combination with cytotoxic 


\section{Case Reports in Gastroenterology}

Case Rep Gastroenterol 2018;12:729-736

DOI: 10.1159/000494751

(c) 2018 The Author(s). Published by S. Karger AG, Basel www.karger.com/crg

Yeatman et al.: A Stage IV Colon Cancer with Extraordinary Response

chemotherapies, has prolonged the survival with metastatic CRC [2-6]. The first-line therapy is generally a combination therapy of FOLFOX (5-fluorouracil, leucovorin, oxaliplatin)/bevacizumab versus FOLFIRI (5-fluorouracil, leucovorin, irinotecan)/bevacizumab; occasionally, cetuximab/panitumumab is substituted in the first line. While cetuximab and panitumumab are anti-EGFR monoclonal antibodies, bevacizumab is a monoclonal antibody targeting VEGF-A, a vascular endothelial growth factor that mediates angiogenesis. Unfortunately, unlike antiEGFR therapies, there are no proven biomarkers that predict response to anti-VEGF therapies [2-4].

Whereas many stage IV/metastatic patients respond to initial therapy, all generally recur over time and succumb to their disease. Moreover, patients with porta hepatis lymph node disease have long been considered incurable, and this has been a well-established contraindication to surgical resection [7-10]. For example, a meta-analysis including 9 studies on a total of 926 CRC patients showed that the prevalence of nodal metastasis after hepatic lymphadenectomy was $16.3 \%$ [11]. The overall 3- and 5-year survival rates of node-negative patients were 53.9\% (424/787) and 32.1\% (246/767), respectively [11]. By contrast, the overall 3and 5-year survival rates of node-positive patients were strikingly lower, namely, $11.3 \%$ $(9 / 151)$ and $1.5 \%$ (2/137), respectively [11]. Thus, understanding the molecular mechanism underpinning the rare, complete pathologic responses may be useful to better treat this disease in the future, particularly as more therapeutic options emerge.

\section{Case Report}

This is the report on a 58-year-old male patient with no previous history of cancer who presented with hematochezia. Whereas he had had a negative colonoscopy 4 years prior, a repeat colonoscopy revealed a mid-transverse, infiltrative, and ulcerated, partially obstructive mass involving $2 / 3$ of the lumen's circumference and measuring $4 \mathrm{~cm}$ in length. A biopsy demonstrated an invasive, moderately differentiated adenocarcinoma. The patient was taking daily enteric coated aspirin at the time of diagnosis to prevent colon cancer and heart disease.

The patient underwent a preoperative computed tomography (CT) scan of the chest, abdomen, and pelvis (Fig. 1a), as well as a preoperative positron emission tomography (PET) scan (Fig. 2a). These scans identified focal thickening of the transverse colon as well as numerous enlarged retroperitoneal and mesenteric lymph nodes. In addition, several hypodense lesions were identified in the right lobe of the liver, the largest measuring $4.2 \mathrm{~cm}$ in diameter. He elected to undergo surgical resection of the primary disease at this time. Resection of the transverse colon cancer and numerous highly enlarged $(>4 \mathrm{~cm})$ and inflammatory lymph nodes, periaortic in location, ensued. Due to the length of the procedure and extensive nodal dissection, as well as encountering an aortic pseudoaneurysm, resection of the liver and porta hepatis lymph node was delayed to a later date.

Final pathology identified a flat, ulcerated, $4.0 \times 3.8 \mathrm{~cm}$ primary lesion in the transverse colon that had perforated the serosa (T4a). Histology showed a moderately differentiated adenocarcinoma. Many lymph nodes contained metastatic disease: 10/22 pericolonic, 1/1 omental, and $9 / 9$ periaortic, with the final stage T4aN2M1. The periaortic lymph nodes were markedly enlarged, desmoplastic, and very adherent to the aorta. Histology was further notable for a papillary architecture in the primary tumor and lymph nodes (Fig. 3); extensive extranodal extension was also observed (Fig. 3b). 
A postoperative PET scan that was performed 2 months after the surgery identified a dominant right hepatic lobe mass as well as porta hepatis adenopathy. All the rest of the retroperitoneal disease had been significantly cleared.

Postoperative therapy was initiated with FOLFIRI/bevacizumab for 4 months. Subsequent scans suggested the patient's disease had disappeared, consistent with a complete clinical response. Subsequent follow-up 4 months later, however, identified recurrence of the same disease. The patient was subsequently retreated once again with FOLFIRI/bevacizumab for 4 months, with a second complete clinical response on PET scan (Fig. 2b). CT scans of the liver also demonstrated calcification of the previous liver metastasis (Fig. 1b). The patient was followed up carefully for $>10$ years with serial CT and PET scans and colonoscopy, and has remained disease free.

Due to the unusual nature of this patient's disease progression and response, he underwent very comprehensive genomic testing. His primary tumor and blood were submitted for whole genome DNA-seq (35×) as well as whole exome RNA-seq (GPS testing; NantHealth, Culver City, CA, USA). This test provides not only identified somatic and germline variants (familial, inherited), but also assesses the gene expression of any called variants. The patient sample also underwent targeted exome sequencing analysis with a panel of $600+$ genes $(1,000 \times)$ (Molecular Health, Houston, TX, USA). Interestingly, despite a different tissue origin for the two genetic tests, there was $100 \%$ concordance among 7 known tumor suppressor and oncogenes in this MSS tumor. In particular, the patient demonstrated concordant mutations in APC (E658*), KRAS $(\mathrm{Q} 61 \mathrm{H})$, and TP53 (Q136*). Other fully concordant mutations included those in EPHA5, KDR, MAP2K4, and PIK3CB. Numerous discordant mutations were identified in both the whole genome sequencing and in the targeted sequencing, consistent with the nonoverlapping data derived from two different tests measuring substantially different numbers of genes. These included JMJD1C, ZNF117, and BRSK1. No germline predisposing variants or fusions were detected. The tumor exonic mutation burden was measured at 113 nonsynonymous mutations or 2.8 variants/Mb. Finally, RNA-seq analysis (gene expression) was used to generate the consensus molecular phenotype assessed to be CMS4 [12].

\section{Discussion}

In the past few years, the introduction of more effective chemotherapeutic agents and biological agents with their promising activities and mild toxicity profiles has pushed the median overall survival time from 12 months to 2 years [2-6]. Currently, bevacizumab, cetuximab, and panitumumab are three biological agents for targeted therapies approved by the FDA for first-line treatment of metastatic CRC [2]. For patients harboring KRAS/NRAS mutations or BRAF (V600E), bevacizumab is the only targeted agent that is used in combination with chemotherapeutic agents. While $>60 \%$ of patients may respond to FOLFOX/FOLFIRI regimens with bevacizumab, no patients are generally cured [2-4]. In addition, no patients with porta hepatis lymphadenopathy survive [7-10]. Here we report an unusual case of a stage IV CRC patient with porta hepatis lymph nodes who responded extremely well to FOLFIRI/bevacizumab with $>10$ years of disease-free survival.

The patient had an APC/KRAS/TP53 (AKP) "triple mutant" genotype. Of note, our recent gene mutation classification of $468 \mathrm{CRCs}$ revealed a prognostic role for $A P C$ that is related to the number of alleles mutated and to the association with other mutant genes such as KRAS and TP53 [13]. The AKP triple mutant was strongly associated with MSS (microsatellite stable) tumors and distant metastasis, and generally conferred a poor prognosis [13]. The patient's 
tumor was found to be also associated with the CMS4 phenotype [12]. Recently, an international consortium has coalesced 6 independent (gene expression) classification systems of CRCs into 4 consensus molecular subtypes: CMS1 (MSI immune), CMS2 (canonical, with strong WNT and MYC activation), CMS3 (metabolic), and CMS4 (mesenchymal), as well as a 5 th unclassified group [12]. CMS4 featured stromal infiltration, TGF- $\beta$ activation, and angiogenesis [12]. A survival analysis indicated that while CMS1 was associated with worse survival after relapse, CMS4 conferred worse overall survival and relapse-free survival [12]. Although CMS4 tumors were associated with increased angiogenesis, treatment of metastatic CRC of this subtype with combination therapies including bevacizumab (an antiangiogenic agent) was reported to show worse outcome [14, 15]. Thus, neither the AKP genotype nor the CMS4 subtype appears to explain the unusually good response of this particular patient to FOLFIRI/bevacizumab therapies as far as bevacizumab is concerned. On the other hand, the patient's tumors were shown to have a papillary architecture (Fig. 3). It was previously reported that VEGF-A was overexpressed in invasive micropapillary colorectal carcinoma [16]. This might contribute to a response to bevacizumab, which is a monoclonal antibody against VEGA-A. In addition, it was recently reported that in first-line chemotherapies, irinotecanbased chemotherapy was significantly superior to oxaliplatin-based chemotherapy for both the progression-free survival and overall survival of patients with CMS4 [17]. This might also partially have contributed to our patient's response, since FOLFIRI/bevacizumab includes irinotecan as a component.

In conclusion, complete, durable, pathologic responses to chemotherapy for metastatic CRC are extremely rare. Here we reported the case of a patient with advanced metastatic disease who has enjoyed a long-term cure with a combination of noncurative surgery and systemic chemotherapy (FOLFIRI)/bevacizumab. The retrospective genetic analysis of the patient's tumor revealed poor-prognosis AKP triple mutant disease. The gene expression analysis suggested that the patient suffered from the CMS4 "immune/angiogenic" class, which was, intriguingly, negatively associated with bevacizumab therapy but positively with irinotecanbased chemotherapy. The histological data suggest the papillary phenotype may be amenable to bevacizumab therapy. Finally, the enlarged lymph nodes suggest a strong immune response despite a low measured tumor mutation burden, which may have ultimately led to the patient's long-term vitality. The deep sequencing analysis has provided some "hints" regarding the molecular mechanisms underpinning this case, and its favorable outcome.

\section{Statement of Ethics}

The authors have no ethical conflicts to disclose.

\section{Disclosure Statement}

The authors have no conflicts of interest to declare.

\section{Author Contributions}

All authors analyzed and interpreted the data; T.J.Y. and M.Y. wrote the manuscript; T.J.Y. conceived the hypothesis and supervised the project. 


\section{References}

1 Siegel RL, Miller KD, Jemal A. Cancer statistics, 2016. CA Cancer J Clin. 2016 Jan-Feb;66(1):7-30.

2 Mahipal A, Grothey A. Role of Biologics in First-Line Treatment of Colorectal Cancer. J Oncol Pract. 2016 Dec;12(12):1219-28.

3 Ohhara Y, Fukuda N, Takeuchi S, Honma R, Shimizu Y, Kinoshita I, et al. Role of targeted therapy in metastatic colorectal cancer. World J Gastrointest Oncol. 2016 Sep;8(9):642-55.

4 Peeters M, Price T. Biologic therapies in the metastatic colorectal cancer treatment continuum-applying current evidence to clinical practice. Cancer Treat Rev. 2012 Aug;38(5):397-406.

5 Yang M, Yeatman TJ. Molecular stratification of colorectal cancer populations and its use in directing precision medicine. Expert Rev Precis Med Drug Dev. 2017;2(4):205-15.

6 Venook AP, Niedzwiecki D, Lenz HJ, Innocenti F, Fruth B, Meyerhardt JA, et al. Effect of First-Line Chemotherapy Combined With Cetuximab or Bevacizumab on Overall Survival in Patients With KRAS WildType Advanced or Metastatic Colorectal Cancer: A Randomized Clinical Trial. JAMA. 2017 Jun;317(23):2392-401.

7 Rodgers MS, McCall JL. Surgery for colorectal liver metastases with hepatic lymph node involvement: a systematic review. Br J Surg. 2000 Sep;87(9):1142-55.

8 Abdalla EK, Adam R, Bilchik AJ, Jaeck D, Vauthey JN, Mahvi D. Improving resectability of hepatic colorectal metastases: expert consensus statement. Ann Surg Oncol. 2006 Oct;13(10):1271-80.

9 Laurent C, Sa Cunha A, Rullier E, Smith D, Rullier A, Saric J. Impact of microscopic hepatic lymph node involvement on survival after resection of colorectal liver metastasis. J Am Coll Surg. 2004 Jun;198(6): 884-91.

10 Jaeck D, Nakano H, Bachellier P, Inoue K, Weber JC, Oussoultzoglou E, et al. Significance of hepatic pedicle lymph node involvement in patients with colorectal liver metastases: a prospective study. Ann Surg Oncol. 2002 Jun; $9(5): 430-8$.

11 Gurusamy KS, Imber C, Davidson BR. Management of the hepatic lymph nodes during resection of liver metastases from colorectal cancer: a systematic review. HPB Surg. 2008;2008:684150.

12 Guinney J, Dienstmann R, Wang X, de Reyniès A, Schlicker A, Soneson C, et al. The consensus molecular subtypes of colorectal cancer. Nat Med. 2015 Nov;21(11):1350-6.

13 Schell MJ, Yang M, Teer JK, Lo FY, Madan A, Coppola D, et al. A multigene mutation classification of 468 colorectal cancers reveals a prognostic role for APC. Nat Commun. 2016 Jun; 7:11743.

14 Stintzing S, Wirapati P, Lenz H-J, Neureiter D, Weikersthal LFv, Decker T, et al. Consensus molecular subgroups (CMS) of colorectal cancer (CRC) and first-line efficacy of FOLFIRI plus cetuximab or bevacizumab in the FIRE3 (AIO KRK-0306) trial. J Clin Oncol. 2017;35(15_suppl):3510-3510.

15 Mooi J, Wirapati P, Asher R, Lee C, Savas P, Price TJ, et al. 4790 - Consensus molecular subtypes (CMS) as predictors of benefit from bevacizumab in first line treatment of metastatic colorectal cancer: Retrospective analysis of the MAX clinical trial. Ann Oncol. 2017;28(suppl_5):v158-v208.

16 Rosa M, Abdelbaqi M, Bui KM, Nasir A, Bui MM, Shibata D, et al. Overexpression of Vascular Endothelial Growth Factor A in Invasive Micropapillary Colorectal Carcinoma. Cancer Contr. 2015 Apr;22(2):206-10.

17 Okita A, Takahashi S, Ouchi K, Inoue M, Watanabe M, Endo M, et al. Consensus molecular subtypes classification of colorectal cancer as a predictive factor for chemotherapeutic efficacy against metastatic colorectal cancer. Oncotarget. 2018 Apr;9(27):18698-711. 


\section{Case Reports in Gastroenterology
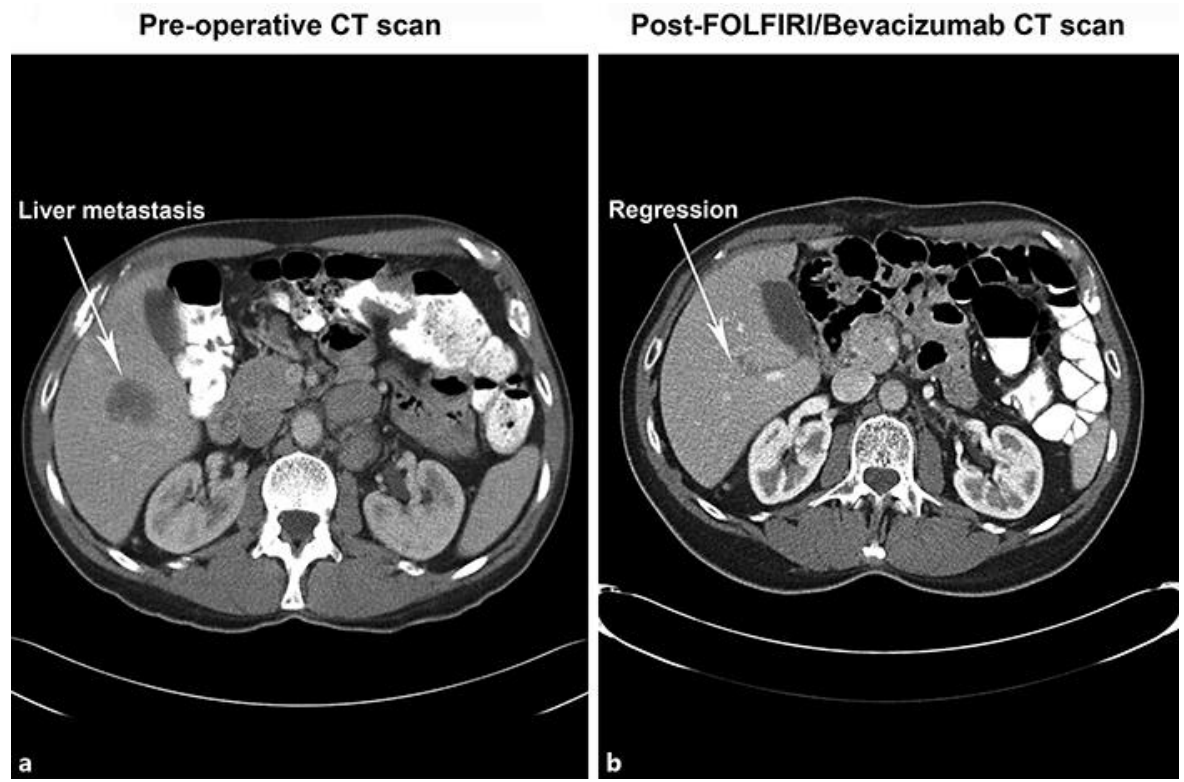

Fig. 1. Computed tomography (CT) scans of the patient. a Preoperative CT scan. b Post-FOLFIRI/bevacizumab CT scan. The CT scans of the liver demonstrated calcification of the previous liver metastasis (arrow) after the therapies.

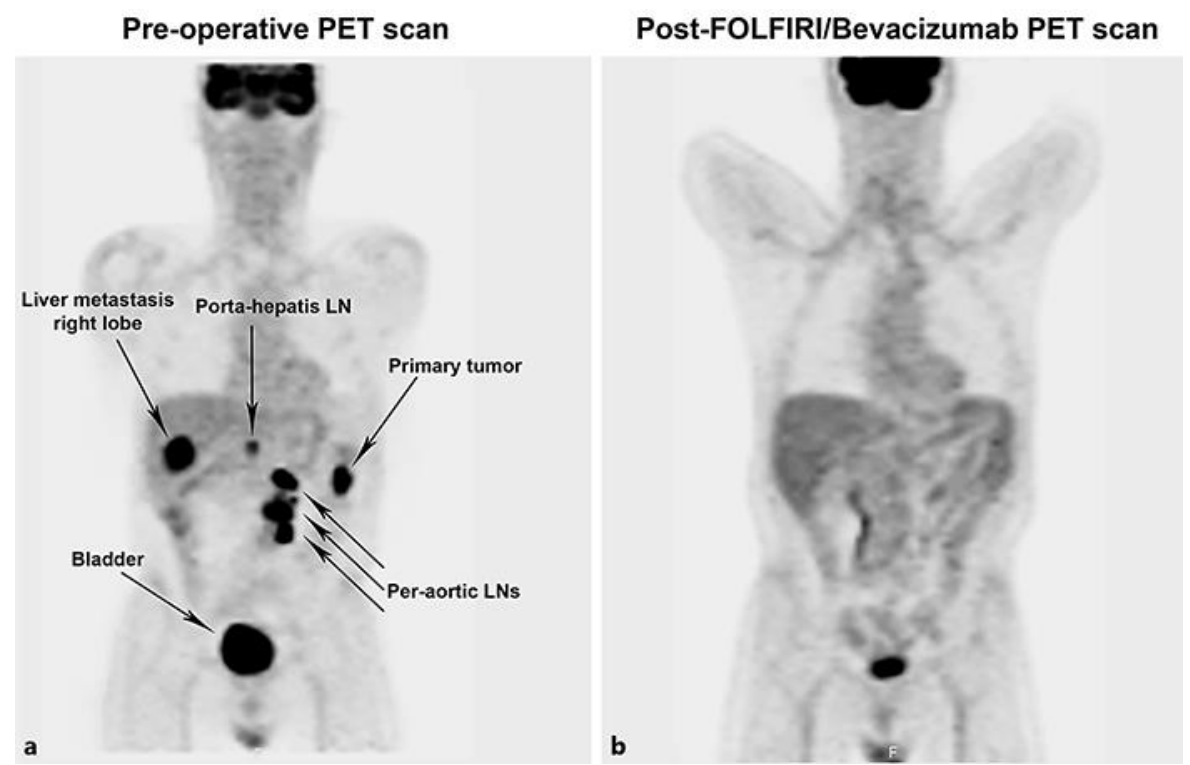

Fig. 2. Positron emission tomography (PET) scans of the patient. a Preoperative PET scan. b PostFOLFIRI/bevacizumab PET scan. Arrows indicate liver metastasis (right lobe), the porta hepatis LN, the primary tumor, periaortic LNs, and the bladder, respectively. The post-therapy PET scan showed that the patient was disease free. $\mathrm{LN}$, lymph node. 


\begin{tabular}{|c|c|c|}
\hline \multirow{2}{*}{$\begin{array}{l}\text { Case Reports in } \\
\text { Gastroenterology }\end{array}$} & \multicolumn{2}{|c|}{ Case Rep Gastroenterol 2018;12:729-736 } \\
\hline & DOI: 10.1159/000494751 & $\begin{array}{l}\text { O } 2018 \text { The Author(s). Published by S. Karger AG, Basel } \\
\text { www.karger.com/crg }\end{array}$ \\
\hline
\end{tabular}

\section{Primary transverse colon tumor}

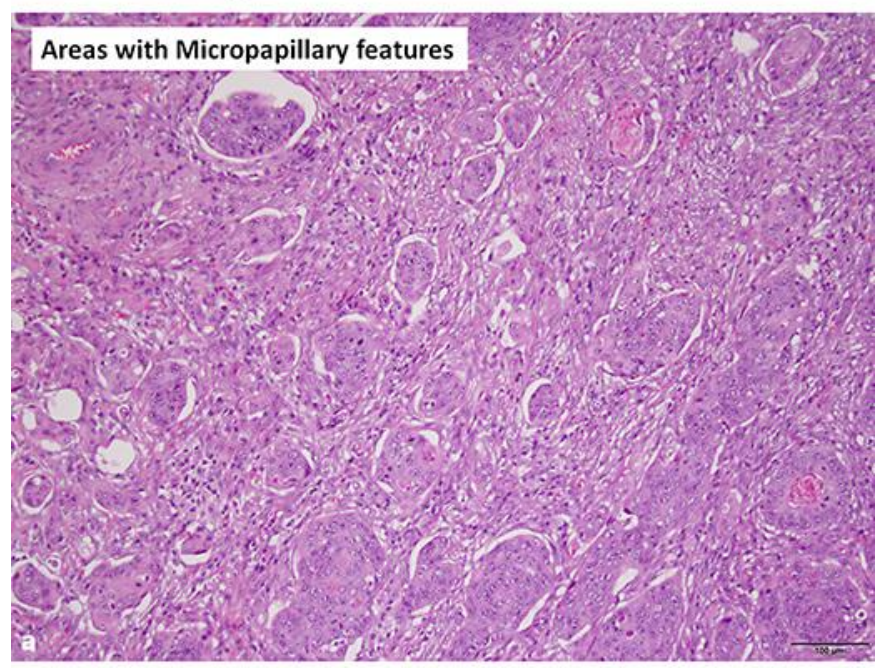

\section{Superior periaortic node}

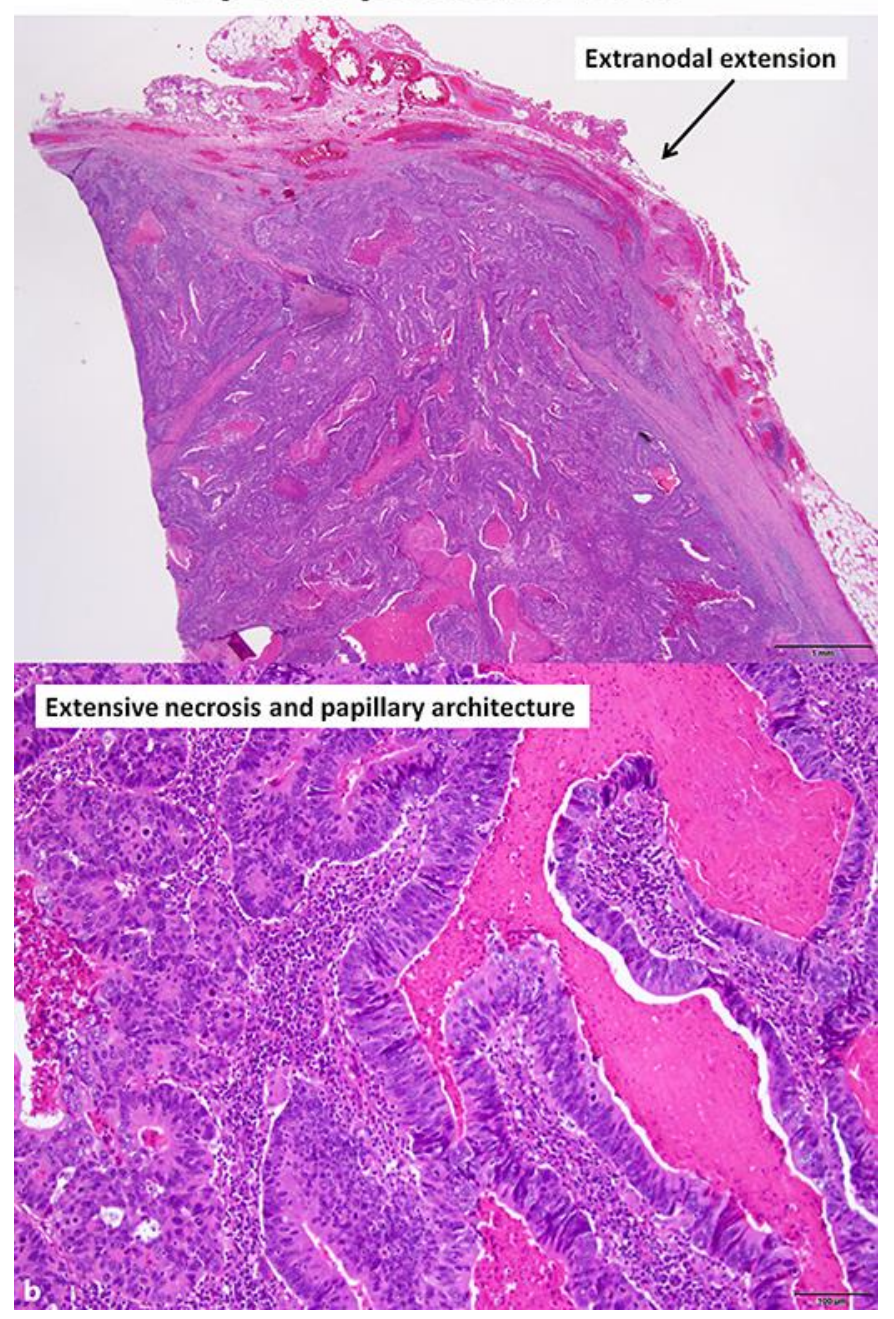




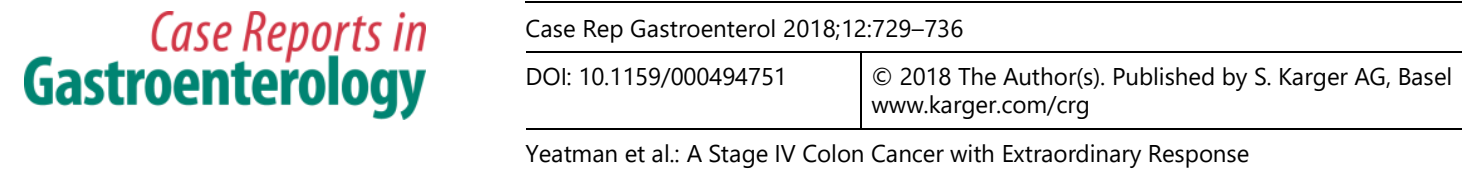

Fig. 3. Histology of the patient. a Primary transverse colon tumor with a papillary architecture. H\&E stain. $\times 200$. b Superior periaortic node with extensive extranodal extension (top panel; H\&E stain; $\times 25$ ) and extensive necrosis and papillary architecture (bottom panel; H\&E stain; $\times 200$ ). 